\section{The long road to determine microplastic trends in a Baltic Sea catchment}

Prof. Dr Matthias Labrenz Leibniz Institute for Baltic Sea Research Warnemünde (10W) Germany

The behaviour and accumulation of microplastics (MPs) in the marine environment has been studied for years but is still poorly understood. In this context, the interdisciplinary MicroCatch_Balt consortium, consisting of environmental scientists, microbiologists, modellers, computer scientists, and ocean managers, is investigating the significance of land-based inputs as a source of MPs into the ocean.

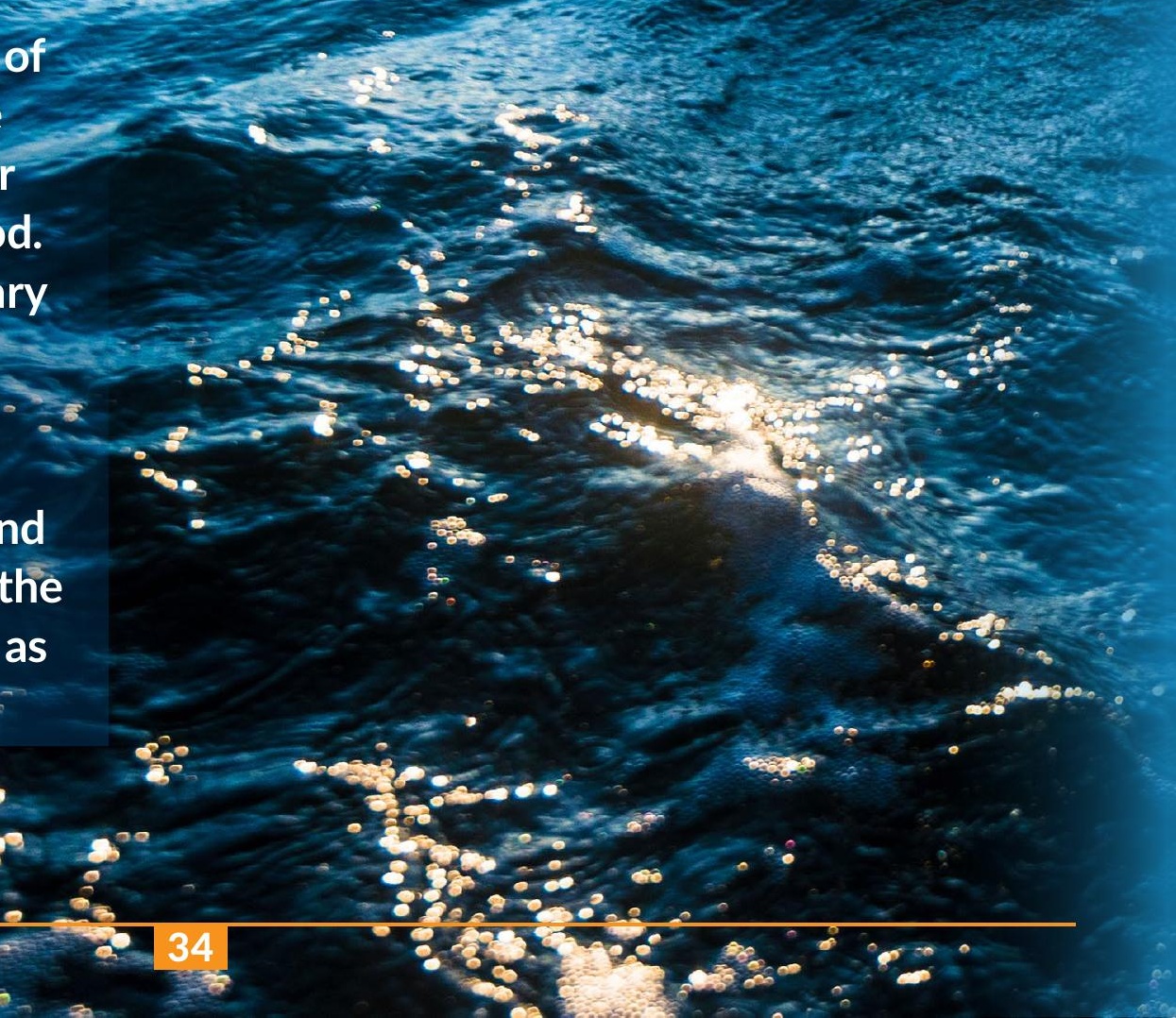

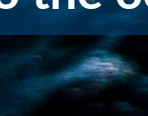

s.
MicroCatch_Balt focuses on the catchment area of the Warnow, the second-largest German freshwater in Baltic Sea inflow. It includes a wide
variety of land uses and integrates a variety of land uses and integrates
high population density with industria high population density with industrial and agricultural areas (Figure 1). Thus, this river catchment can be considered representative
Baltic Sea.

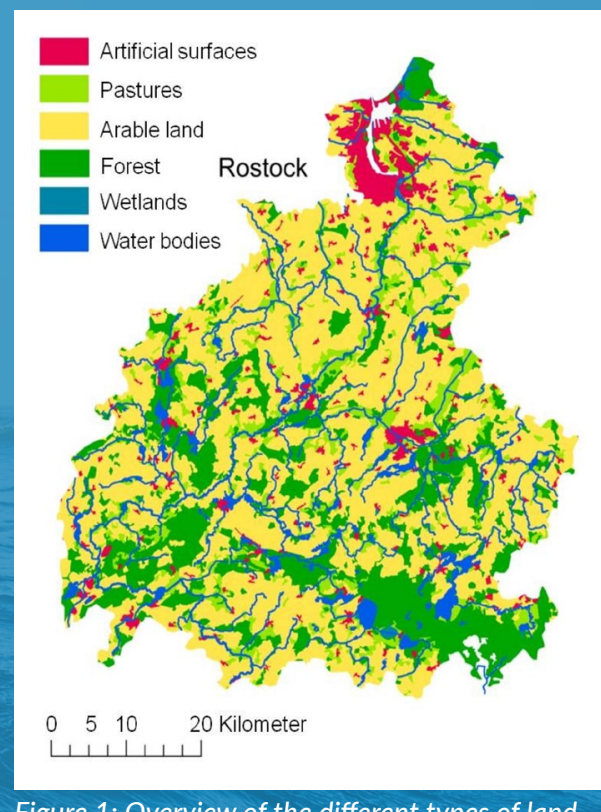

Figure 1: Overview of the different types of land
use in the Warnow river catchment area (modified use in the Warnow river catchment area (moc
from CORINE Land Cover (CLC2006) by Inga
Krämer).

Sampling, processing and analysis

Most of the MicroCatch_Balt partners already had prior experience with the Warnow catchment-not only in the field of MPs (Labrenz, 2017) but also in modelling the Warnow catchment, its estuary and the Baltic Sea itself. Nevertheless, it quickly became clear: during the project that fundamental optimisations and new developments sampling processing and analys to obtain meaningful results for MP modelling In principle the identification of MPs from the environment was fartoo for labour htensive and time consuming to the mods. Not specific to Microced Balt, this is a limiting factor worldwide.
For example, it turned out that net-based methods for sampling MPs in water are inadequate for the study area. These nets usually have mesh sizes of about 100$300 \mu \mathrm{m}$. Comparable to most aquatic systems, however, MPs in the Warnow and the Baltic Sea are predominantly smaller, are not caught by nets and are lost to analysis. For this reason, MicroCatch_Balt developed the mobile sampling device 'Rocket' (Figure 2).

Based on filtration cartridges, this device captures all particles $>10 \mu \mathrm{m}$ (Lenz and Labrenz, 2018), a first and essential advance for the evaluation of MPs in the aquatic environment. The Rocket allows sampling of about $1 \mathrm{~m}^{3}$ of water, depending on water quality. A second crucial step was to increase the volume of Warnow water to be investigated identifying MPs from the drinking water treatment sludge of the Rostock water treatment sludge of the Rostock treatment plant intakes surface water from the Warnow river and purifies it by coagulation/flocculation and filtration processes. From the MPs contained in the accruing treatment sludge, MP derived in an integrative way, as the sampling covers a period of at least three hours of flow in the Warnow river. Both methods independently estimated MP concentrations in the lower part of the Upper Warnow amount to $\sim 200$ particles $/ \mathrm{m}^{3}$ of water (Siegel et al., 2021).

Time is the critical factor in MPs processing. Therefore, MicroCatch Balt also tested new procedures for work processes. Sediments/soils have a high content of natural organic and inorganic matter and thus, have to be cleaned in a complex way. By developing and applying electrostatic separation, especially for MPs Succeeded signtring MPs method allows the removal of significant amounts of natural contaminations before further processing of the MPs,

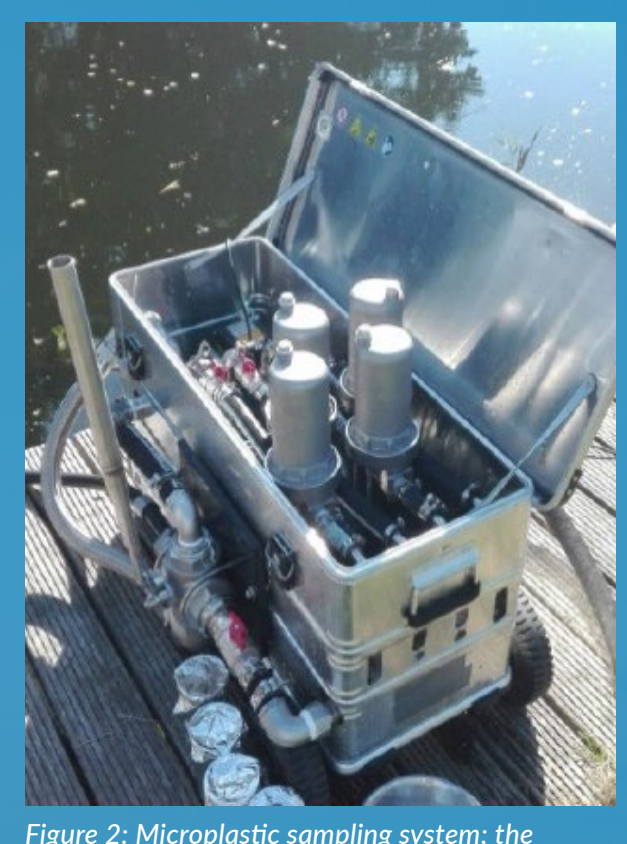

(the

that MP purification steps could be implified (Enders et al., 2020a).

The subsequent processing of MPs fom the environment was also fundamentally improved. To enable MP identification via Raman/FT-IR MP greatly reduced without harming elastics themselves. This is possible the enzymatic and oxidative digestion. The enzymatic approach is gentle but very technical and time-consuming. However, it remained unclear whether $\mathrm{MP},<100 \mu \mathrm{m}$ in particular, might be modified or impaired by the faster oxidative treatment. MicroCatch_Balt was able to prove that this is not the case and that chemical treatment can et al., 2021).

The final identification of MPs from the environment was an anticipated challenge from the beginning. MP particles cannot be reliably identified y the eye or with purely microscopic mothods. For this purpose, Raman/FTR spectroscopy, as already mentioned, re adequate methods. Again, the procedures were very time-consuming, nd the identification of MP particles MPs pipensiderable bottleneck in the MPs pipeline. 
Gepard Enabled PARticle Detection

MicroCatch_Balt provided the scientific community with a self-developed, opensource software package allowing particle analysis using Raman microspectroscopy.

The GEPARD (Gepard Enabled PARticle Detection) software enables the acquisition of an optical image, then detects particles and uses this information to drive the spectroscopic measurement. This results in a multitude of possibilities to efficiently check, correct and report all obtained results (Brandt et al., 2020). The final implementation to reduce measurement time is to refine total sample numbers by using representative
subsamples. However, these must still provide reliable results.

\section{Identification and}

quantification

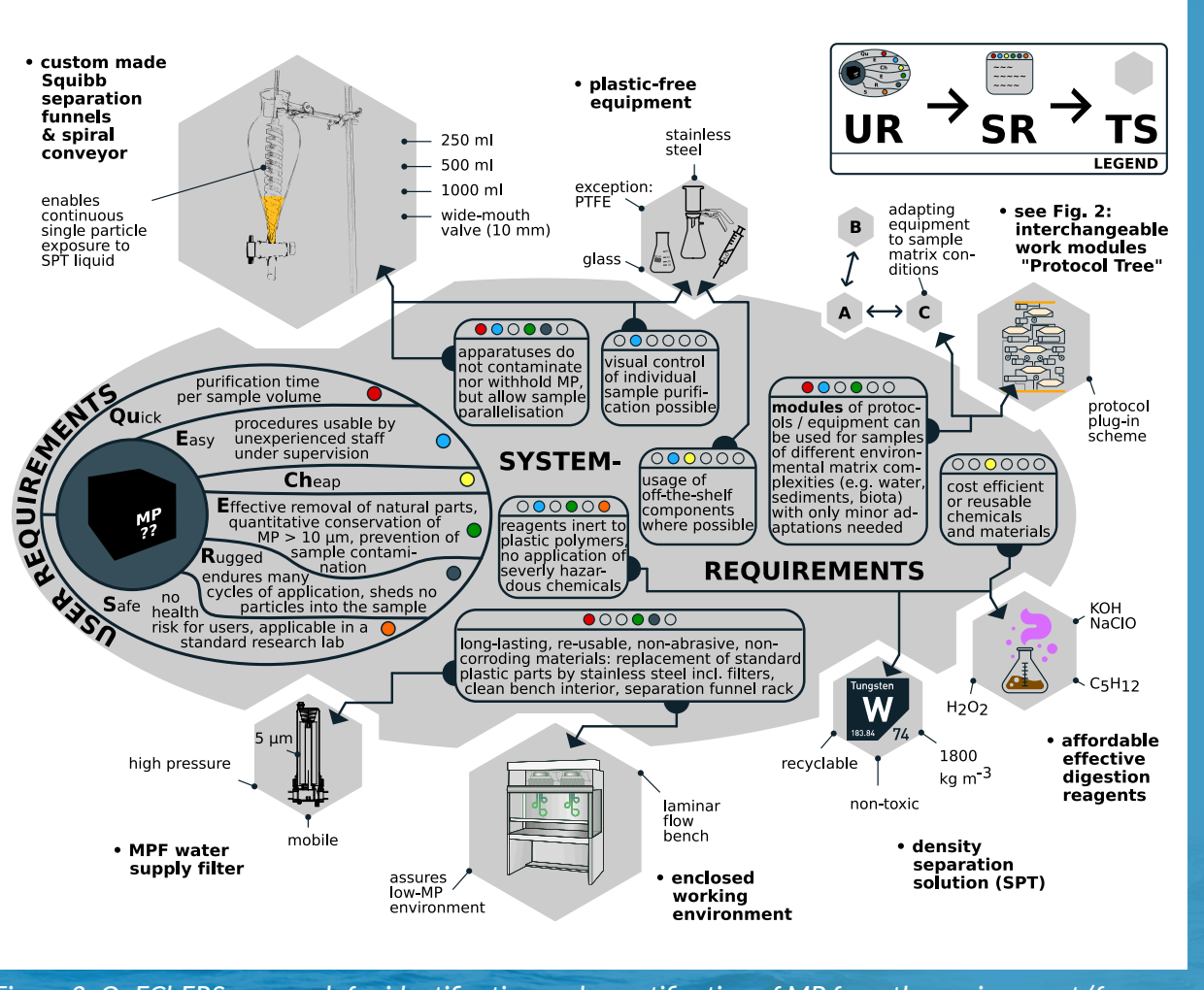

Figure 3: QuEChERS approach for identification and quantification of MP from the environment (from

MicroCatch_Balt comprehensively evaluated different proposed subsampling methods on aselection of real samples from different environmental compartments. It was shown that subsampling errors are largely due to statistical countin errors. However, mainly by increasing the proportion of MP particles in the samples, the subsampling error can be kept low (Brandt et al., 2021).

Thus, MicroCatch_Balt has developed an optimised and highly reproducible pipelin to identify and quantify MPs from the environment. This has been incorporated
into a universal framework of modular protocols that both meet predefined user requirements ('QuEChERS': quick, eas, cheap, effective, rugged and safe) and provide best practices for appropriat working conditions in a standard laboratory, available to the public since 2020 (Enders et al., 2020b)

MicroCatch_Balt has also provided, for the first time, a modelling system that can map the complete pathway of MPs principle and monitor the effectiveness

\section{of mitigation measures with 'what-if' Conclusion} core element of an exhibition travelling catchment $(0.53 \mathrm{~g} / \mathrm{haMP})$. The even be identified yet (Tagg and Labrenz, scenarios. Concurrently, this powerful tool forms the basis for the application of a But what have the MicroCatch Balt multi-touch table that provides interactive investigations now revealed in concrete learning materials. It now serves as the terms for the situation in the Warnow Stralsund to Flensburg and has already been requested nationwide. Everyone is guaranteed access to the MicroCatch Balt online app [https://microcatch.
ostseeforschung.info/en/start.html ostseeforschung.info/en/start.htm (Figure 4)]

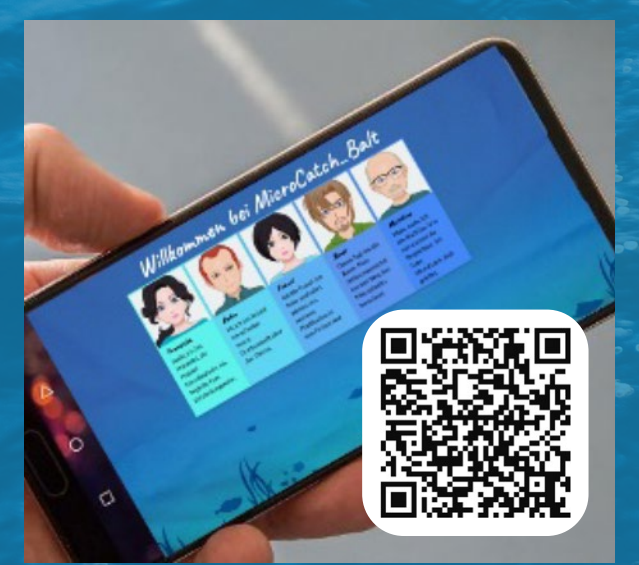
catchment area?

Based on the mathematical simulation model RAUMIS, it can be said that sewage sludge is the most important agricultural MPs source in the Warnow application of sewage sludge increases the soil load from 4 to $15 \mathrm{MP}$ particles/g. The mGROWA-TeMBa model system was developed to represent runoff modelling in the Warnow and from urban areas. Emissions from diffuse and point sources (atmospheric deposition, sewage sludge, wastewater treatment plants, etc.) were considered. Other pathways may not 2018).

Although MP concentrations in the river are highly variable, it is clear that urban emissions dominate the Warnow along the German Baltic Sea coast from $\begin{array}{lll}\text { Figure 4: MicroCatch_Balt online app (Foto: Sven Hille).". } & \text { urban emissions dominate the Warnow } & \text { Tagg, A. S. and Labrenz, M. (2018) 'Closing microplastic pathways before they open: A mod. } \\ \text { estuary; } 49.4 \text { per cent of MP inputs come } & \text { Environmental Science and Technology, 52, pp.3340-3341. doi: 10.1021/acs.est.8b00961. }\end{array}$ from the catchment, and 43.1 per cent Ocean, do not exist in the Baltic Sea. It from stormwateroverflows. The Rostock Ocen, do not exist in the Baltic Sea. It only 1.4 per cent. Therefore, measures stormwater runoff and emissions from
the combined sewer system (Piehl et al. the combined sewer system (Piehl et al. 2021).

MicroCatch_Balt can estimate that the annual input of MPs from the Warnow into the Baltic Sea is $152-291$ billion particles (mainly 10-100 $\mu \mathrm{m}$ in size) (Piehl et al., 2021). However, a majority of the particles are already washed ashore near the point of emission. Beaches are the main accumulation areas with up to $10^{9} \mathrm{MP} / \mathrm{m}^{2} / \mathrm{a}$. The residence time of MPs after emission into the Baltic Sea is 14 days on average (Schernewski et al., reduce MPs via beach clean-up. However, this alone is no solution.

It also became clear that high amounts of MPs do not even reach the Baltic Sea but remain in the sediment of the Warnow (Enders et al., 2019).

Finally, the MicroCatch_Balt investigations also revealed that MPs are not degraded/assimilated by microorganisms in the Warnow or Baltic Sea but simply continue to fragment (Oberbeckmann and Labrenz, 2020), consequently continuing accumulation in distinct ecosystems. Thus, the solution to the environmental problem is a simple 14 first place.

the sub-equatorial oyres of the Atlantic

\section{References}

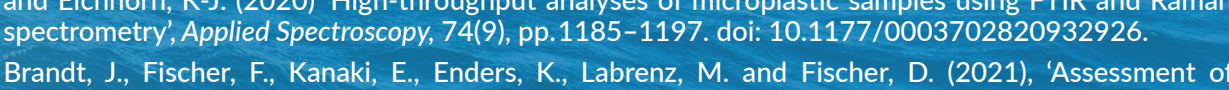

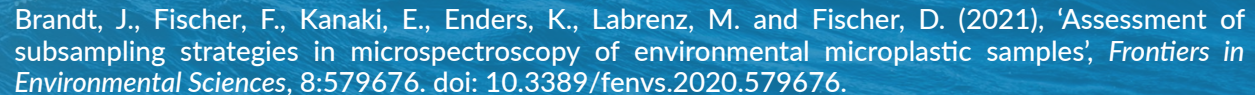

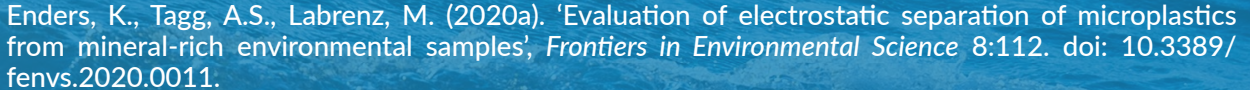

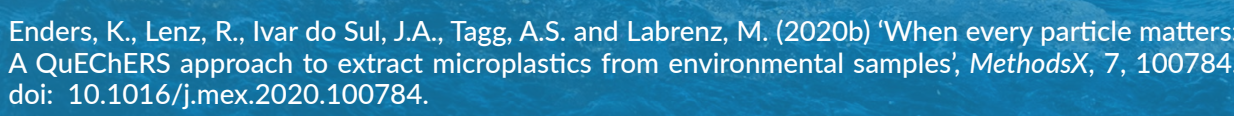

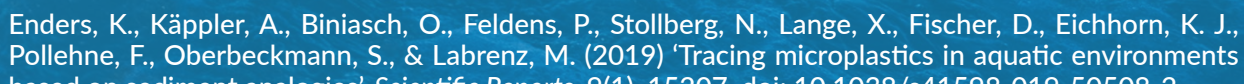
15207. doi: 10.1038/s41598-019-50508-2.

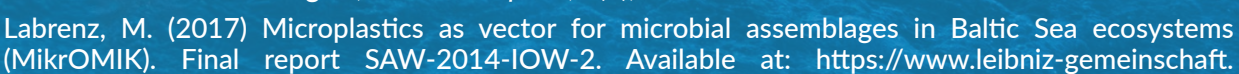
Lenz, R., Labrenz, M. (2018) 'Small microplastic sampling in

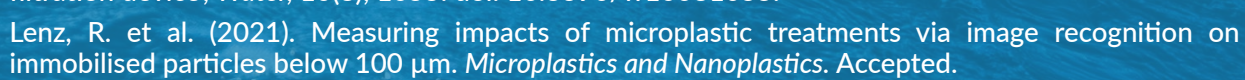

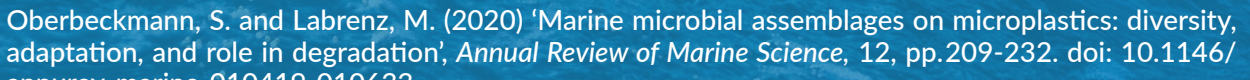

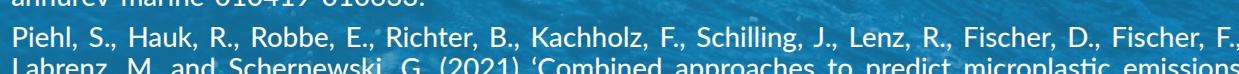

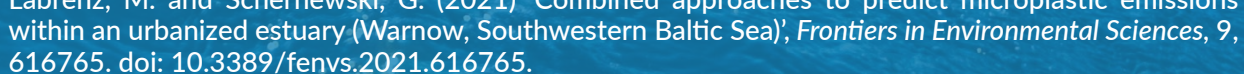

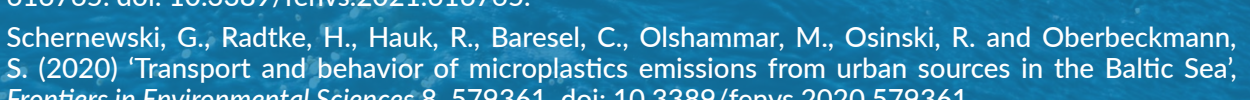

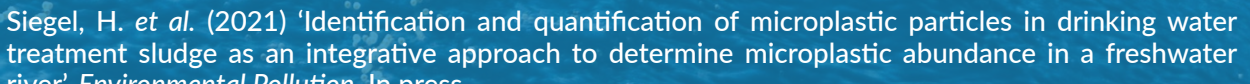

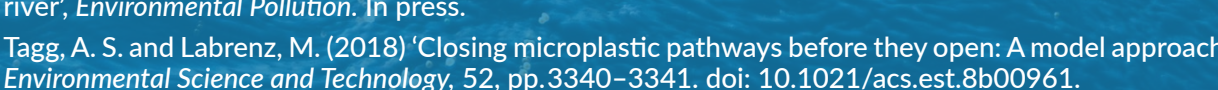
wastewater treatment plant contributes $\begin{aligned} & \text { even seems possible to quantitatively } \\ & \text { res via beach clean-up. However, }\end{aligned}$ to reduce MP inputs should focus on one: do not introduce MPs there in the

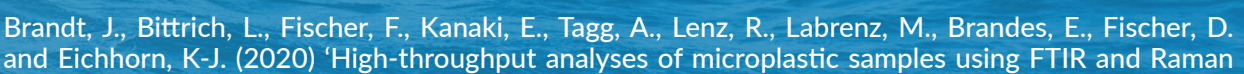
def/lileadinin/user-upload/Bilder-und.DDownloads/Forschung/Wettbewerb/Vorhaben/

One System: Analysing Microplastics Sinks area to the open Baltic (MicroCatch_Balt)

PROJECT SUMMARY

The multidisciplinary MicroCatch_Balt project determines inland sources and sinks processes on its pathways to the open Baltic Sea within the exemplary Warnow catchment area. It covers the most significant aspects of microplastic contamination in Northern Germany-from limnic to marine systemsproviding expertise to support stakeholders in designing future monitoring or mitigation strategies.

PROJECT LEAD Prof. Labrenz received a PhD in natural
sciences at the University of Kiel (1999) and worked as a postdocin thic USA and Germany. His main research focus is the role of microorganisms in anthropogenic influenced
marine systems. He currently teaches as marine systems. He currentily teaches
Professor of Environmental Microbiology at the University of Rostock (Germany) and Es Associate Professor of Microorganisms

\section{PROJECT PARTNERS}

The network project MicroCatch_Balt is ed by the Leibniz Institute for Baltic Sea partner institutes are the Leibniz Institute of Polymer Research Dresden (IPF), Fraunhofer Institute for Computer Graphics Research IGD, Forschungszentrum Juelich, and

\section{CONTACT DETAILS}

Prof. Dr Matthias Labrenz Warnemünde (IOW)

Seestrasse 15

18119 Rostock-Warnemuende Germany

黑 +49(0)3815197378

$\square$ matthias.labrenz@io-warnemuende.de

(D) www.io-warnemuende.de/ environmental-microbiology.html

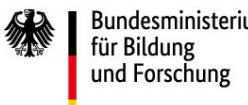

FUNDING

Funding for this study was received from the
German Federal Ministry of Education and Research German Federal Ministry of Education and Researc
(BMBF) under grant agreement No.03F0788A. 